Usages et représentations du français hors de France

\title{
Une histoire des origines
}

\author{
Daniel Coste
}

\section{(2) OpenEdition}

Journals

Édition électronique

URL : https://journals.openedition.org/dhfles/3660

DOI : $10.4000 /$ dhfles.3660

ISSN : 2221-4038

Éditeur

Société Internationale pour l'Histoire du Français Langue Étrangère ou Seconde

Édition imprimée

Date de publication : 1 janvier 2013

ISSN : 0992-7654

Référence électronique

Daniel Coste, «Une histoire des origines », Documents pour l'histoire du français langue étrangère ou seconde [En ligne], 50 | 2013, mis en ligne le 01 janvier 2017, consulté le 28 mars 2023. URL : http:// journals.openedition.org/dhfles/3660 ; DOI : https://doi.org/10.4000/dhfles.3660

Ce document a été généré automatiquement le 28 mars 2023.

Tous droits réservés 


\title{
Une histoire des origines
}

\author{
Daniel Coste
}

1 Il y a quelque paradoxe à ce que, pour une société travaillant dans le domaine de l'histoire, les origines de sa fondation ne soient documentées que de manière lacunaire. Certes, la date de l'assemblée constituante de la SIHFLES ne souffre pas de doute. Mais, de cette réunion générale, nul compte rendu analytique disponible. Et le présent signataire, qui fut aussi le premier président de la société, a donc scrupule à indiquer d'emblée que les traces font défaut de la réunion fondatrice et que, comme pour toute histoire des origines, la scène primitive reste en partie mythique ${ }^{1}$. Cette séance tenue il y a vingt-cinq ans, demandera toutefois qu'on y revienne pour ouvrir une série de rappels ordonnés ici sous quatre intitulés: un lieu de mémoire; un homme d'affiliations ; un moment pour l'histoire ; un champ en construction.

\section{Un lieu de mémoire}

2 Ce lieu est celui même où a trouvé place la manifestation marquant le $25^{\mathrm{e}}$ anniversaire de la société, à savoir la Grande bibliothèque du Centre international d'études pédagogiques de Sèvres (désormais CIEP). Le 5 décembre 1987, une cinquantaine de personnes s'y réunissent, ayant répondu à une invitation du BELC (Bureau pour l'enseignement de la langue et de la civilisation françaises à l'étranger), du CIEP, de la revue Le français dans le monde (désormais FDLM), signée respectivement par André Reboullet, ancien directeur-adjoint du BELC et ancien rédacteur en chef de la revue, Francis Debyser, directeur-adjoint du CIEP et ancien directeur du BELC, Jacques Pécheur, rédacteur en chef du FDLM.

Entre autres participants à cette séance, des collègues étrangers: Carla Pellandra (université de Bologne), Elisabet Hammar (université de Stockholm), Herbert Christ (université de Giessen), Konrad Schröder (université d'Augsbourg), tous quatre invités par Reboullet en raison de leurs travaux sur l'histoire de l'enseignement du français dans leurs pays respectifs ${ }^{2}$. Mais, pour Schröder, c'est aussi, plus largement, d'histoire de l'enseignement des langues étrangères (et pas seulement du français) qu'il devrait s'agir, et il le fait valoir dans la discussion relative aux statuts de la future société. Paul 
Gerbod, historien des relations culturelles et spécialiste du XIX ${ }^{\mathrm{e}}$ siècle, André Chervel, spécialiste du français et historien de l'éducation, membre de l'équipe de Pierre Caspard à l'INRP' ${ }^{3}$, sont présents, ainsi que Jean-Claude Garreta, directeur de la bibliothèque de l'Arsenal. Nombre des autres participants français appartiennent au domaine du français langue étrangère, mais on manque d'une liste des présents.

4 L'assemblée de décembre avait été précédée d'une réunion préparatoire en juin 1987, au CIEP déjà, réunion « qui a rassemblé des historiens de la littérature, de la langue, de l'éducation, de la culture, des sociologues, des didacticiens, des responsables de revues» (extrait d'une notice dans la revue Reflet, $\mathrm{n}^{\circ}$ 22, p. 60). Et, dans un article programmatique paru dans le $\mathrm{n}^{\circ} 208$ du FDLM sous le titre «Pour une histoire de l'enseignement du F.L.E. », Reboullet (1987a) avait en quelque sorte précadré les ambitions et le projet d'action de l'association à venir.

Comment nommer l'association ? L'intitulé final a été précédé de quelques autres essais qui ne sont pas indifférents quant aux options. Reboullet pensait d'abord à «Association internationale pour l'histoire de l'enseignement du français à l'étranger ». Et l'assemblée constituante balance entre "association » et "société », entre "français à l'étranger " et "français langue étrangère ", entre "français langue étrangère » et « français langue étrangère ou seconde ", avant de s'arrêter au titre et au sigle que l'on connaît.

Lieu inaugural, le CIEP l'est aussi, entre autres événements, par l'assemblée constituante, en 1968, de l'Association française des professeurs de français (AFPF, rebaptisée ensuite AFEF, Association française des enseignants de français), qui concerne avant tout le français langue maternelle et qui deviendra, en 1969, membre de la Fédération internationale des professeurs de français (FIPF), alors tout juste créée. Le rappel que la Grande bibliothèque du CIEP avait vu cette autre fondation n'est pas vraiment éloigné du présent propos dans la mesure où, parmi les initiateurs de l'AFPF, se trouve déjà Reboullet, qui en sera le premier secrétaire général et, jusqu'en 1972, le rédacteur en chef de la revue Le français aujourd'hui ${ }^{4}$.

7 En bref, dans cette Grande bibliothèque du CIEP, lieu de mémoire à bien des titres et pour bien des acteurs du monde de l'éducation et de la coopération éducative internationale, l'entrepreneur Reboullet n'en est plus tout à fait, en ce mois de décembre 1987, à son coup d'essai.

\section{Un homme d'affiliations}

8 Homme d'associations, Reboullet est d'abord homme d'affiliations, et cela depuis la création de la revue FDLM en 1961. Pour lui, le projet initial d'une telle revue est bien de mettre en relation les professeurs de français à travers le monde. Il aurait voulu en faire non pas tant un instrument de diffusion culturelle et didactique à partir d'un centre qu'un lieu partagé de parole et d'écrit des enseignants eux-mêmes, pour affilier ces derniers et faire entendre leur diversité afin de mieux promouvoir ce qui les unit. Mais FDLM dépend pour bonne part des commandes du ministère des Affaires étrangères et la revue, constitutivement liée au BELC, entretient aussi des rapports étroits avec le CRÉDIF (Centre de recherche et d'étude pour la diffusion du français, École normale supérieure de Saint-Cloud). Reboullet ne peut guère se passer du concours rédactionnel de ces centres de recherche et de formation, mais se méfie des méthodologies présentées comme nouvelles et susceptibles de se durcir en 
théorisations prescriptives. Tout ce qui ressemble à une doxa lui est étranger ${ }^{5}$. Lui qui est au centre souhaiterait faire jouer la périphérie, s'appuie aussi sur l'Alliance française, autre réseau d'affiliations, et maintient de bonne relations avec Marc Blancpain, longtemps secrétaire général de l'Alliance et, comme lui, ancien de l'ENS de Saint-Cloud. En tout état de cause, à l'épreuve, Reboullet doit bien constater qu'il est difficile de susciter des contributions suffisamment diversifiées des enseignants de français étrangers, tant sont variables les conditions de travail d'un pays à l'autre, les moyens à disposition, les expériences de l'écrit.

9 Mais Reboullet est tenace. Le projet qui verra naître la FIPF (cf. ci-dessus, note 4) est pour lui une autre manière de créer du lien et de multiplier et réunir - en principe - des initiatives locales et une dynamique plus polycentrique. Ce n'est pas non plus un hasard si, au moment de quitter la rédaction de la revue FDLM en 1981, il publie le dernier numéro thématique spécial qu'il aura commandé et qui, sous le titre « Des professeurs de français » (Le français dans le monde, 1981) cherche à rendre compte d'une pluralité de témoignages d'enseignants de tous pays et de tous statuts.

Il est permis de penser que, faute d'avoir pu donner vraiment la parole aux enseignants à la tâche dans ce vaste espace qu'est censée couvrir une revue du français dans le monde, Reboullet verra dans la création de la SIHFLES un moyen de ressusciter les voix oubliées des maitres du passé et de relativiser ainsi les modes dominantes du court terme.

11 En somme, dans la trajectoire personnelle de Reboullet, la SIHFLES n'a rien d'un hapax. Elle répond, sous une nouvelle forme, à une visée ancienne poursuivie avec constance et détermination depuis la conception du FDLM, à travers l'AFPF, la FIPF, voire la première esquisse du dictionnaire pour le professeur de français.

12 Rassembler, mettre en contact, combiner le lien dans l'espace et la filiation dans le temps... et cela, bien au-delà de la fondation de la SIHFLES : dans les dossiers du bureau de Reboullet, figuraient encore, après son décès, des notes manuscrites et des correspondances (en particulier avec Willem Frijhoff) relatives à l'ambitieux projet d'une sorte d'histoire générale des enseignants de français hors de France ${ }^{6}$.

Dans le projet qu'il dresse en 1987 pour la société naissante (Reboullet 1987; Besse 2012), Reboullet brasse large et entend que l'on s'intéresse aux contextes, aux publics, aux marchés économiques, aux enjeux institutionnels et politiques, aux diverses catégories d'acteurs ; mais on remarquera que ses propres travaux et contributions, en particulier dans Documents, portent essentiellement sur des figures d'individus, maîtres de langues célèbres ou non, petits agents d'une grande aventure historique.

14 L'originalité de la SIHFLES par rapport aux initiatives antérieures tient bien sûr à ce qu'il s'agit cette fois de recherche ${ }^{7}$ et que la dimension internationale des participants actifs se situe au niveau universitaire et de manière de plus en plus polycentrée. Non plus mettre en lien des enseignants mais constituer un réseau de chercheurs et les rassembler sur un agenda ambitieux : faire connaitre ce qui existe ici ou là et combler peu à peu des lacunes dans la connaissance de ce passé multiforme. L'œuvre de Ferdinand Brunot est restée inachevée, le prolongement que lui a donné Charles Bruneau n'apporte rien à l'exploration du français du dehors. À bien des égards, nombre d'études ponctuelles paraissent ne présenter qu'un intérêt monographique, voire anecdotique ou hagiographique. Bref, il n'est guère difficile de réunir à Sèvres pour le lancement d'une société savante, quelques-uns du petit nombre des chercheurs 
étrangers d'envergure qui, par leurs acquis et par leurs apports, peuvent animer l'œuvre collective dont le programme est tracé, selon les trois axes prolongeant l'œuvre de Brunot : aspects didactiques, aspects linguistiques, aspects sociologiques de l'histoire de ce français du dehors.

Avec une certaine austérité et rigueur qu'on dirait volontiers - cliché oblige protestante, Reboullet le rassembleur ne s'est jamais posé en leader ; en 1987, à 71 ans, il n'entend pas présider l'association qu'il a suscitée. Non pas homme de l'ombre certes, mais médiateur, initiateur, instigateur. Le paradoxe dernier reste que cet homme d'affiliation n'était pas un homme de réunion, un amateur de rassemblements physiques. Il participera très peu aux conseils d'administration, aux assemblées générales et même aux colloques de la SIHFLES. Mi statue du commandeur, mi grandpère ronchon, il ne manquera toutefois pas de se manifester fréquemment par des courriers incitatifs et aiguillonnants. Avant de prendre, l'âge venu, une distance plus résolue, il donnera l'impression de considérer que la société qu'il avait imaginée et fait naître n'était pas tout à fait à la hauteur de ses espérances, manquant d'ambition dans son développement et dans la véritable construction d'un projet de recherche international. Sans doute n'était-il pas assez conscient de ce qu'une société de ce type, même savante et même s'agissant du français, ne peut guère mobiliser de gros bataillons de chercheurs et de doctorants dans un domaine où, même chez les historiens de plein exercice, la reconnaissance universitaire demeure plus que limitée.

\section{Un moment pour l'histoire}

16 Tout ceci, en France même, ne se passe évidemment pas dans un espace clos ni n'advient du seul fait de la volonté d'un homme. L'histoire est, si l'on peut dire, dans l'air du temps.

171974 avait vu la publication, sous la direction de Jacques Le Goff et de Pierre Nora, des trois volumes de Faire de l'histoire. Nouveaux problèmes. Nouvelles approches. Nouveaux objets. Le projet de la SIHFLES pourra, un peu plus d'une dizaine d'années plus tard s'inscrire modestement dans telle ou telle de ces nouveautés. Au cours de ces années 1970 et 1980, histoire et sociologie sont en train de prendre le pas sur la linguistique comme disciplines de référence dans le domaine des sciences humaines et sociales et se renouvellent en effet dans leurs méthodes et leurs objets. Les deux premiers tomes de la série Les lieux de mémoire, dirigée par Pierre Nora, paraissent respectivement en 1984 (La République) et en 1986 (La Nation).

18 À l'INRP, autour de Caspard, le Service d'histoire de l'éducation a été créé en 1977. On a noté plus haut que Chervel, Alain Choppin, Gérard Bodé, chercheurs dans ce Service, suivront de façon intéressée les travaux de la SIHFLES.

Dans le domaine même de l'enseignement du français aux étrangers et de la politique de diffusion de la langue et de la culture, divers anniversaires ont donné lieu à publications. La Mission laïque française marque ses 80 ans en 1982 par un volume, Éléments pour une histoire de la Mission laïque française (Bancilhon 1982). En 1983, c'est l'Alliance française qui célèbre son centenaire sous le titre L'Alliance française. Histoire d'une institution (Bruézière 1983). Toujours en 1983, Albert Salon publie L'action culturelle de la France dans le monde, ouvrage issu d'une thèse à dimension historique (Salon, 1983). À l'occasion du centenaire de l'ENS de Saint-Cloud, une table ronde organisée en 1982 à propos de la diffusion du français après 1945, donne lieu à la parution du volume 
Aspects d'une politique de diffusion du français langue étrangère depuis 1945. Matériaux pour une histoire (Coste (dir.) 1984). Deux thèses d'État ont été soutenues en 1987, qui intéressent directement l'histoire récente du français langue étrangère : celle de Sophie Moirand, portant sur les discours de la revue FDLM et leur évolution (Moirand, 1988) ; celle de Daniel Coste sur la période 1945-1975 et les relations entre linguistique appliquée et français langue étrangère (Coste 1987) ${ }^{8}$. Quant à l'ouvrage - qui fera date de Christian Puren, Histoire des méthodologies de l'enseignement des langues, il sera publié en 1988 (Puren 1998, [1988]).

Dans ce mouvement d'intérêt pour l'histoire plus ou moins contemporaine et à visée plus ou moins réflexive, les travaux scientifiques voisinent avec le commémoratif, l'hagiographique, le récit de vie, mais l'ensemble manifeste bien un goût pour la rétrospection et, sinon toujours de nouveaux problèmes, du moins des approches en partie nouvelles pour de nouveaux objets.

21 Mais si les initiatives prises par Reboullet s'inscrivent à certains égards dans cette mouvance générale, elles ne sauraient s'en tenir à des formes d'histoire à chaud non exemptes du risque de courte vue. À propos des travaux relatifs au français langue étrangère dans sa période la plus récente, Reboullet exerce un humour un peu grinçant : " Histoire certes, mais une histoire dont la période couverte est courte et le champ surtout hexagonal. Nous attendions Braudel et Duby, nous trouvons Lacouture » (Reboullet 1987 : 56).

\section{Un champ en constitution}

Promouvoir un projet d'histoire du français langue étrangère ou seconde n'a pas qu'à voir avec une montée en puissance des sciences historiques. Le français langue étrangère (bien plus que le français langue seconde) s'établit peu à peu dans le champ institutionnel des disciplines et de la reconnaissance universitaire. Chevalier et Encrevé (cf. ci-dessus note 8) connaissent bien les travaux de Pierre Bourdieu et leur contribution à une histoire sociale des sciences du langage se situent indirectement dans ce cadre. C'est aussi en lecteur attentif de Bourdieu que Porcher, qui a été directeur du CRÉDIF et est en 1982 conseiller scientifique du directeur de la Coopération et des Relations internationales au ministère de l'Éducation nationale, impulse, après avoir été rapporteur d'une commission présidée par le recteur Auba (on retrouve le CIEP), la création de filières de français langue étrangère (FLE) : maitrise et mention de licence dans les universités (Porcher, 1986). Le même Porcher avait créé en 1985 l'ASDIFLE (Association de didactique du français langue étrangère).

En 1986, Robert Galisson et Porcher dirigent un numéro de la revue Études de Linguistique Appliquée, titré "Priorité(s) FLE », mais où Galisson présente et défend sa conception d'une didactologie des langues et des cultures (Galisson, 1986) et manifeste ainsi une certaine tension, dans la constitution institutionnelle du champ, entre Français langue étrangère et Didactique des langues (Coste 2013). Les débats, au moment de la création de la SIHFLES quant à l'extension du territoire à explorer (histoire du français langue étrangère et/ou seconde ou, plus largement, des langues, selon le choix de Schröder) ne sont pas sans rapport avec ces problèmes plus généraux de positionnement.

24 Et c'est en 1987, sur ces questionnements, que le CRÉDIF organise le colloque «Didactique des langues ou didactiques de langues. Transversalités et spécificités », 
dont le maître d'œuvre est Denis Lehmann et qui réunit des didacticiens et linguistes de français langue étrangère, de langues étrangères autres et de français langue maternelle9.

En bref, sur quelques années de la décennie 1980, le FLE renforce sa constitution en champ institutionnalisé non seulement autour des centres historiques comme le CRÉDIF ou le BELC ou de la revue LFDM, mais surtout avec la mise en place de filières universitaires de FLE (et non de didactique des langues) et d'associations "dédiées ", comme l'ASDIFLE et la SIHFLES.

Sans surprise, c'est le français langue étrangère "hexagonal » qui est massivement représenté dans le premier bureau de la SIHFLES :

Président: Daniel Coste (CRÉDIF, ENS de Saint-Cloud); vice-présidents: André Reboullet (ex BELC et FDLM) et Louis Porcher (UFR de didactique du FLE de l'université Paris 3 et ex CRÉDIF); secrétaire général : Claude Olivieri (BELC); secrétaire général adjoint: Jacques Pécheur (BELC et LFDM); trésorière-adjointe : Gisèle Kahn (CRÉDIF). Seuls, le trésorier, Roland Desné (spécialiste de la littérature du XVIII ${ }^{e}$ siècle) et les deux autres vice-présidents, Jean-Claude Chevalier et Herbert Christ, présentent des profils différents.

Quant au premier conseil d'administration, fort de 26 membres (dont 23 Français), il ne compte que 5 femmes (Marie-Hélène Clavères, Elisabet Hammar, Gisèle Kahn, Carla Pellandra, Geneviève Zarate).

Un quart de siècle plus tard, en 2012, sur les 29 membres du conseil (dont 4 appartenaient déjà au premier conseil de 1987), 16 sont des femmes et 18 se trouvent hors de France ; 6 (dont 3 retraités) relèvent du domaine de la didactique française du FLE. Sur 10 membres du bureau, 4 sont des femmes, 7 se trouvent hors de France, 2 sont didacticiens "hexagonaux» du FLE. Et sur les 7 président-e-s qui se sont succédé depuis 1987 (dont 2 femmes), seuls 2 sont français et seul le premier relevait du groupe des didacticiens français.

Cet énoncé de chiffres serait fastidieux, s'il ne faisait apparaître qu'en 25 ans la SIHFLES, au niveau de ses instances statutaires, a su s'internationaliser et se féminiser plus fortement, tout en se dégageant de l'emprise initiale des tenants de la didactique institutionnelle et "hexagonale» du FLE. Il y aurait là quelques commentaires possibles, mais qui tiennent à une autre histoire...

31 Un lieu, un moment, un champ, de multiples facteurs ont sans doute, autant ou plus que l'action d'un homme, joué à l'origine de la SIHFLES. C'est ce qu'on a essayé de rappeler ici à grands traits. Disons que, voici 25 ans, les temps étaient venus. Encore fallait-il que quelqu'un les accouche.

BIBLIOGRAPHIE

BANCILHON, Nicole (1982). Éléments pour une histoire de la Mission laïque française. 1902-1982. Paris : Mission laïque française. 
BESSE, Henri (2012). «L'équipée de la SIHFLES ». Brochure pour le 25e anniversaire. CIEP, Sèvres, 14 décembre 2012, 45-61.

BRUÉZIÈRE, Maurice (1983). L'Alliance française. Histoire d'une institution. Paris : Hachette.

CHEVALIER, Jean-Claude \& ENCREVÉ, Pierre (dir.) (1984). « Vers une histoire sociale de la linguistique ». Langue française, 63.

CHEVALIER, Jean-Claude avec ENCREVÉ, Pierre (2006). Combats pour la linguistique, de Martinet à Kristeva. Essai de dramaturgie épistémologique. Lyon : ENS Éditions.

CHRIST, Herbert (1988). « Pour une histoire sociale de l'enseignement du français ». Documents pour l'histoire du français langue étrangère ou seconde, 1, 6-10.

COSTE, Daniel (1987). Institution du français langue étrangère et implication de la linguistique appliquée. Contribution à l'étude des relations entre linguistique et didactique des langues de 1945 à 1975 . Thèse d'État, université Paris 8, sous la direction de Jean-Claude Chevalier.

COSTE, Daniel (dir.) (1984). Aspects d'une politique de diffusion du français langue étrangère depuis 1945. Matériaux pour une histoire. Paris : Hatier.

COSTE, Daniel (2013). « La didactique des langues entre pôles d'attraction et lignes de fracture ». In Jean-Claude Beacco (dir.) Éthique et politique en didactique des langues. Autour de la notion de responsabilité. Paris : Didier, coll. Langues et didactique.

DABÈNE, Michel (2008). « Quelques repères, perspectives et propositions pour une didactique du français dans tous ses états ». In Jean-Louis Chiss, Jacques David \& Yves Reuter (dir.). Didactique du français. Fondements d'une discipline. Bruxelles : De Boeck.

GALISSON, Robert \& COSTE, Daniel (dir.) (1976). Dictionnaire de didactique des langues. Paris : Hachette.

GALISSON, Robert \& PORCHER, Louis (dir.) (1986). « Priorité(s) FLE ». Études de Linguistique Appliquée, 64. Paris : Didier Érudition.

GALISSON, Robert (1986). «Éloge de la 'Didactologie/didactique des langues et des cultures (maternelles et étrangères)' ». Études de Linguistique Appliquée, 64, 39-54.

HAMMAR, Elisabet (1988). « Sur un manuel suédois de français publié au XVIIIe siècle ». Documents pour l'histoire du français langue étrangère ou seconde, 1, 11-16.

Le français dans le monde (1981). « Des professeurs de français ». Le français dans le monde, 161.

LE GOFF, Jacques \& NORA, Pierre (dir.) (1974). Faire de l'histoire. Nouveaux problèmes. Nouvelles approches. Nouveaux objets. Paris : Gallimard.

MOIRAND, Sophie (1988). Une histoire de discours... une analyse des discours de la revue « Le Français dans le monde »1961-1981. Paris : Hachette.

NORA, Pierre (dir.) $(1984,1986)$. Lieux de mémoire, tome 1 : La République, tome 2 : La Nation. Paris : Gallimard.

PORCHER, Louis (1986). « Priorités institutionnelles », Études de Linguistique Appliquée. 64, 75-92.

PUREN, Christian (1988). Histoire des méthodologies de l'enseignement des langues. Paris : Nathan CLE International.

REBOULLET, André (1987). « Pour une histoire de l'enseignement du F.L.E. ». Le français dans le monde, 208, 56-60. 
REBOULLET, André \& FICHOUX-REBOULLET, Yvonne (1987). Six générations de Vivarois et de Bretons. Brochure.

SALON, Albert (1982). L'action culturelle de la France dans le monde. Paris : Nathan.

\section{NOTES}

1. Gisèle Kahn, Évelyne Argaud et Gérard Vigner n'ont retrouvé que quelques notes dans les archives de la société et des copies de notices annonçant ou suivant la création de la SIHFLES. Je tiens à les remercier pour la communication de ces documents, dont certains présents dans la bibliothèque personnelle d'André Reboullet, léguée à la SIHFLES pour les pièces la concernant.

2. Elisabet Hammar et Herbert Christ présentent à cette occasion des exposés qui seront publiés dans le $\mathrm{n}^{\circ} 1$ de Documents pour l'histoire du français langue étrangère ou seconde, en juin 1988.

3. Reboullet avait établi des contacts avec Pierre Caspard, et d'autres chercheurs de cette équipe, tels Alain Choppin et Gérard Bodé, participeront activement à la SIHFLES.

4. Si Reboullet s'est intéressé d'aussi près à la création et à la mise en œuvre de l'AFPF, c'est aussi qu'avec le Belge Louis Philippart, président de la Société belge des professeurs de français, et avec l'appui bienveillant du recteur Jean Auba, directeur du CIEP, il animait ce projet de création d'une fédération internationale des professeurs de français et que, jusqu'en 1968, les professeurs de français langue maternelle ne disposaient pas d'une association susceptible de faire partie d'une telle fédération. On peut aussi noter que, dans le premier bureau de l'AFPF, présidée par Pierre Barbéris, balzacien enseignant à l'École normale supérieure de Saint-Cloud, figurent aussi Jean-Claude Chevalier et Daniel Coste, que l'on retrouve, vingt ans plus tard, avec Reboullet, dans le premier bureau de la SIHFLES.

5. Autre source peut-être de déception, malgré la réussite durable de l'entreprise : Reboullet avait suscité la réalisation d'un Dictionnaire "pour le professeur de français », qui devait rendre accessible à toutes et tous des revues telles que FDLM et être un vecteur de langage commun. L'élaboration fut essentiellement le fait de membres du BELC et du CRÉDIF et, quand l'ouvrage paraît enfin, en 1976, il s'intitule Dictionnaire de didactique des langues (Galisson \& Coste (dir.) 1976) et constitue au moins autant un geste d'affirmation d'un nouveau domaine disciplinaire qu'un instrument pratique au service des enseignants « de terrain ».

6. Affiliation donc, mais aussi filiation dans la durée. L'année même de création de la SIHFLES, Reboullet publie avec son épouse, à compte d'auteur, une brochure, Six générations de Vivarois et de Bretons (Reboullet \& Fichoux-Reboullet 1987), où sont rétablies les lignées et les parentés dont elle, bretonne et lui-même, ardéchois, font partie. Exercice de généalogie à la fois régionale et sociale, où il s'agit de marquer des inscriptions dans des contextes historiques locaux ou bien des parcours d'ascension professionnelle ou encore des effets de mobilité géographique.

7. Le choix académique d'une désignation comme "société " plutôt que « association » (même si cela ne change rien au statut de l'instance) est à cet égard significatif.

8. Toujours dans le même temps, Louis Porcher engage des entretiens avec des acteurs du domaine à propos de leur parcours et de leur vision du domaine : Reboullet et Philippe Greffet (alors secrétaire général de l'Alliance française) sont ainsi enregistrés. Le projet aurait dû être poursuivi par la SIHFLES, mais n'aura pas comme tel de suite, ce que l'on peut regretter. Ces entretiens-témoignages sont autant de ressources pour un travail socio-historique. Chevalier et Pierre Encrevé y ont recours pour leur projet d'histoire sociale de la linguistique (Chevalier \& Encrevé, 1984 ; Chevalier avec Encrevé, 2006).

9. Ces contacts interdidactiques n'aboutiront pas à de véritables rapprochements entre les langues comme le donne aussi à voir la création d'associations différentes à vocation commune de regroupement de chercheurs ( $c f$. Dabène, 2008). L'association DFLM (Association pour le 
développement de la recherche en didactique du français langue maternelle) est fondée en 1986 (et devient en 2004 l'AIRDF : Association internationale de recherche en didactique du français). L'ACEDLE (Association des chercheurs et enseignants-didacticiens des langues étrangères) date de 1989.

\section{RÉSUMÉS}

L'assemblée constituante de la Société internationale pour l'histoire du français langue étrangère ou seconde (SIHFLES) se tient, en 1987, dans un lieu riche d'histoire, le CIEP de Sèvres. Cette fondation est due en particulier à l'action d'André Reboullet qui est aussi à l'origine d'autres initiatives pour rassembler les enseignants de français. Elle s'inscrit dans une période où les études historiques et commémoratives se multiplient et se renouvellent, et dans un contexte où le champ universitaire et associatif du français langue étrangère se développe.

The meeting which created the Société internationale pour l'histoire du français langue étrangère ou seconde (SIHFLES) took place in 1987 at the CIEP, in France. That creation resulted in part from initiatives taken by André Reboullet, who had already launched several other actions to foster contacts among teachers of French as a foreign language. SIHFLES was founded, at a time of renewal of historical studies in France and of a number of commemorations, in a general context where the field of French as a foreign language was establishing itself firmly in universities and in the associative world.

\section{INDEX}

Keywords : SIHFLES, French as a foreign language, A. Reboullet, associations, teachers of French, 1987.

Mots-clés : SIHFLES, français langue étrangère, A. Reboullet, associations, enseignants de français, 1987.

\section{AUTEUR}

DANIEL COSTE

ENS de Lyon 